\title{
Fetal Response to Intra-Uterine Needling: Is It Pain? Does It Matter?
}

\author{
A review of: Fisk NM, Gitau R, Teixeira JM, et al. 2001 Effect of direct fetal opioid analgesia on fetal hormonal and \\ hemodynamic stress response to intrauterine needling. Anesthesiology 95:828-835
}

$\mathrm{T}^{\prime}$ HE USE OF analgesia prior to invasive procedures in term and preterm neonates has become widely accepted. However, the use of analgesics during procedures on the fetus (ranging from a simple tissue biopsy to more complex surgical repairs for congenital malformations) had remained unstudied.

A group of investigators from the Imperial College of Medicine, London have been able to use a now commonly used clinical procedure of blood transfusions in alloimmunized fetuses to address this question. A standard technique of gaining intravenous access in the fetus is to insert a needle in the umbilical vein at the placental cord insertion (PCI), which has no sensory innervation but there are significant risks of causing cord tamponade or umbilical artery spasm. An alternative technique is to go through the fetal abdomen to insert a needle in the intra-hepatic vein (IHV). This approach may have less risks but is technically more difficult and requires more time to gain access. Additionally, human fetuses respond by vigorous body and breathing movements suggesting that this procedure maybe noxious. Technical factors and ease of approach determine the route of needle insertion. In a series of articles since 1994, Fisk and colleagues have reported that fetuses as young as 18-20 weeks, independent of the maternal responses, show marked elevations in plasma cortisol and -endorphin after more than 10 minutes of IHV needling, but show no responses to the PCI technique $(1,2)$. This stress response is accompanied by fetal hemodynamic changes as measured by a decrease in the middle cerebral artery

\section{Adnan T. Bhutta Sarita Garg Cynthia R. Rovnaghi}

(MCA) pulsatility index in fetuses as young as 16 weeks (3).

In their latest article, Fisk et al investigated whether fentanyl would blunt this stress response in fetuses undergoing blood transfusions between 20-35 weeks (4). As randomization into IHV and PCI groups was constrained by ethical and technical considerations, the group decided to study the fetal effects of a 10 $\mathrm{g} / \mathrm{kg}$ fentanyl single dose by "longitudinal analysis" in 12 fetuses, which underwent one IHV transfusion with fentanyl and one IHV transfusion without. They also performed a "cross-sectional analysis" comparing 16 fetuses that underwent IHV transfusions with fentanyl to the 29 fetuses transfused at the IHV or PCI sites without fentanyl. The pregnancies were uncomplicated except for alloimmune fetal anemia or thrombocytopenia. The investigators found that use of fentanyl was associated with significantly reduced -endorphin levels and MCA pulsatility index values, less than the non-fentanyl IHV group and approaching those of the PCI group.

Previous studies from this group stimulated a vigorous debate in academic circles about whether the stress response shown by the fetus constituted pain. Some scientists feel that fetuses do not feel pain, as they do not have a conscious appreciation of pain (5). Others have argued that fetuses cannot appreciate pain before 26 weeks of gestation as the thalamo-cortical connections are not established before then (6). Yet another group has suggested that fetuses as young as 10 weeks may be able to per- ceive pain and that the thalamus may play a role in this perception (7). The scientific value of this debate has degenerated because of obvious links with the pro-life vs. pro-choice debate.

A more relevant question would be to ask whether the stress response secondary to noxious stimuli can be avoided or treated. Rapid development of the CNS occurs during the second and third trimesters of pregnancy and exposure to noxious stimuli would be expected to cause long-term neurodevelopmental changes (8).

With their collective work, Fisk and colleagues have made a huge contribution to our understanding of fetal physiology and may have spawned the new field of fetal anesthesia.

1. Giannakoulopoulos X, Sepulveda W, Kourtis P, Glover V, Fisk NM 1994 Fetal plasma cortisol and beta-endorphin response to intrauterine needling. [see comments]. Lancet 344:77-81

2. Gitau R, Fisk NM, Teixeira JM, Cameron A, Glover V 2001 Fetal hypothalamic-pituitary-adrenal stress responses to invasive procedures are independent of maternal responses. J Clin Endocrinol Metabol 86:104-109

3. Teixeira JM, Glover V, Fisk, NM 1999 Acute cerebral redistribution in response to invasive procedures in the human fetus. Am J Obst Gynecol 181:1018-1025

4. Fisk NM, Gitau R, Teixeira JM, Giannakoulopoulos $\mathrm{X}$, Cameron AD, Glover VA 2001 Effect of direct fetal opioid analgesia on fetal hormonal and hemodynamic stress response to intrauterine needling. Anesthesiology 95:828- -835

5. Derbyshire SW, Furedi A 1996 Do fetuses feel pain? 'Fetal pain' is a misnomer. [see comments]. BMJ 313:795

6. Lloyd-Thomas AR, Fitzgerald M 1996 Do fetuses feel pain? Reflex responses do not necessarily signify pain. BMJ 313:797-798

7. Saunders PJ 1997 Do fetuses feel pain? We should give them the benefit of the doubt. [letter; comment]. BMJ 314: 303

8. Anand KJ 2000 Pain, plasticity, and premature birth: a prescription for permanent suffering? Nat Med 6:971-973

Arkansas Children's Hospital, S-431

800 Marshall Street

Little Rock, AR 72202, U.S.A.

bhuttaadnant@uams.edu 